\title{
The effects of physics and chemical- abundance uncertainties on the properties of lower-mass stars that are used as standard candles
}

\author{
Don A. VandenBerg \\ Department of Physics \& Astronomy, University of Victoria, P. O. Box 3055, Victoria, \\ B.C. V8W 3P6, Canada \\ email: vandenbe@uvic.ca
}

\begin{abstract}
The distances to old stellar populations have traditionally been derived from three standard candles: the luminosity of the red-giant-branch tip, the absolute magnitudes of RR Lyrae stars, and the colour-magnitude-diagram loci of nearby subdwarfs. The distance-modulus uncertainties that are associated with these methods are still at the level of $\pm 0.10-0.15$ mag. Current stellar models are able to satisfy these age-independent constraints to well within their error bars, which are mainly owing to the uncertainties in the distances of the calibrating objects and, directly or indirectly, to chemical-abundance uncertainties. The impact of varying the physics and the assumed abundances in stellar models on the aforementioned standard candles is discussed, as is the use of the giant-branch bump luminosity to constrain the distance scale.
\end{abstract}

Keywords. Hertzsprung-Russell diagram, stars: distances, stars: evolution, stars: fundamental parameters, subdwarfs

\section{Introduction}

For the most part, our understanding of basic stellar physics (equation of state, nuclear reactions, opacities) has reached the point where further significant improvements are unlikely. Certainly, the biggest revision that was made during the past 10 years was to the rate of the important ${ }^{14} \mathrm{~N}(p, \gamma){ }^{15} \mathrm{O}$ reaction (see, e.g., Formicola et al. 2004; Marta et al. 2008), although notable advances were also made to conductive-opacity data (Cassisi et al. 2007; Itoh et al. 2008) and to low-temperature radiative opacities (Ferguson et al. 2005). However, because all of the physics ingredients have now been carefully examined, it seems unlikely that further major changes to the physics relevant for the deep interiors of stars, in particular, will occur. Indeed, it is fair to say that, insofar as the predicted luminosities of lower-mass $\left(\lesssim 1 \mathrm{M}_{\odot}\right)$ stars are concerned, physics uncertainties are likely less of a problem than those associated with chemical-abundance determinations (see, e.g., VandenBerg et al. 2012, hereafter VBD12).

Unfortunately, the same cannot be said for the model $T_{\text {eff }}$ scale. Although noteworthy improvements have been made to our understanding of the atmospheric boundary condition (VandenBerg et al. 2008), systematic errors in the predicted temperatures of stars in different parts of the Hertzsprung-Russell diagram must be present to some extent, no matter what treatment of the surface boundary conditions is employed. Moreover, most stellar models still use the standard mixing-length theory (MLT; Böhm-Vitense 1958), with a constant value of the free parameter, $\alpha_{\mathrm{MLT}}$, to treat convection, even though there are indications based on 3D model atmospheres that this parameter may vary with temperature and (possibly) with evolutionary state (Trampedach \& Stein 2011). Another 
problem that has ramifications for model temperatures is the current lack of a good theory for the additional mixing that apparently must occur just below surface convection zones to avoid conflicts between the predicted and observed surface abundances of stars (see, e.g., Nordlander et al. 2012; and references therein). This extra mixing, likely caused by turbulence (Richer et al. 2000), counteracts the effects of gravitational settling.

Part of the difficulty in improving these aspects of stellar models is that the empirical $T_{\text {eff }}$ scale is uncertain by $\sim 70-100 \mathrm{~K}$, although encouraging progress has been made on this front by Casagrande et al. (2010). In addition, measured $[\mathrm{Fe} / \mathrm{H}]$ values often vary by as much as $\pm 0.15-0.20$ dex (owing in part to the aforementioned $T_{\text {eff }}$ difficulties). Until the basic properties of stars have been established to much higher accuracy and precision than they are at present, any of the standard candles that require precise temperatures (or colours) and metal abundances, such as local subdwarf calibrators, will always have fairly large uncertainties associated with them. The main purpose of this study is to describe and quantify the effects of current physics and chemical-abundance uncertainties on the properties of lower-mass stars that are used for distance determinations.

\section{The Luminosity of the Red-Giant-Branch Tip}

The absolute $I$-band magnitude of the red-giant-branch (RGB) tip is an especially good standard candle, because it is luminous, nearly independent of mass (and hence age) as well as metallicity (e.g., Da Costa \& Armandroff 1990), and it has a solid physics basis. Moreover, the RGB tip is a ubiquitous and relatively well-defined feature of old, populous stellar systems. One of the best available calibrations of $M_{I}^{\mathrm{RGBT}}$ with metallicity is that derived by Bellazzini et al. (2004) from a careful analysis of near-infrared (NIR) photometry of the globular clusters (GCs) $\omega$ Cen and 47 Tuc. Their calibration is given by the dashed curve in the left-hand panel of Fig. 1, where the two clusters are represented by the filled circles with error bars.

The solid curves illustrate how well BASTI evolutionary calculations (Pietrinferni et al. 2010) are able to reproduce the Bellazzini et al. (2004) results when different

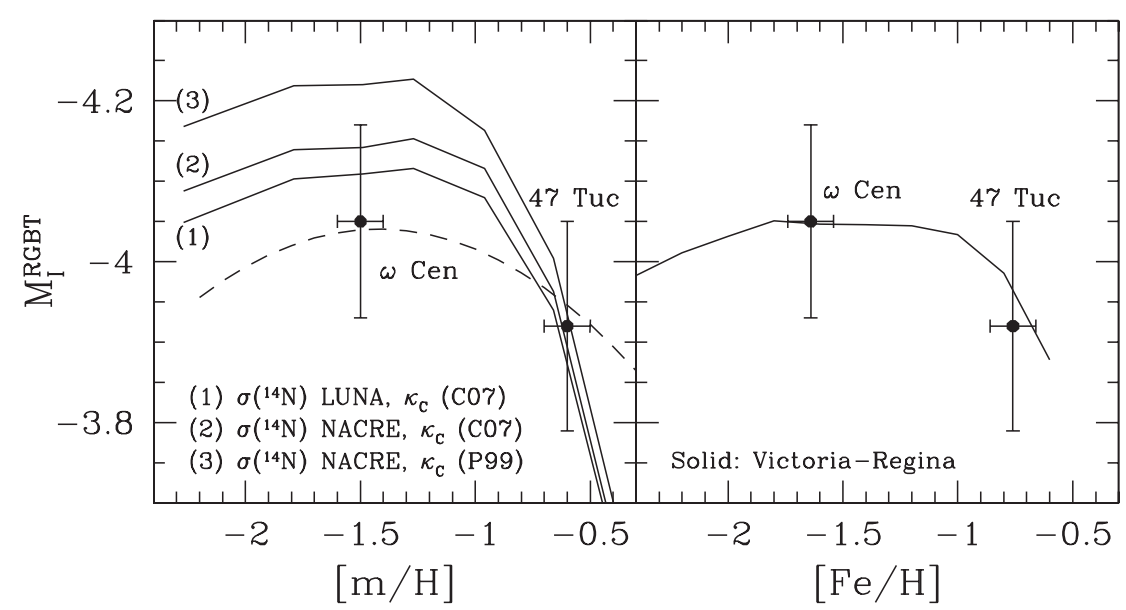

Figure 1. (left) Comparison of the empirical determination (dashed curve and filled circles) of $M_{I}^{\mathrm{RGBT}}$ as a function of $[\mathrm{m} / \mathrm{H}]$ by Bellazzini et al. (2004) with the predictions of BASTI models (Pietrinferni et al. 2010), on the assumption of different rates for the ${ }^{14} \mathrm{~N}(p, \gamma)^{15} \mathrm{O}$ reaction and/or different conductive opacities (see text). (right) The same Bellazzini results for $\omega$ Cen and 47 Tuc, compared with the predictions of Victoria-Regina (VBD12) models for the RGB tip luminosities of $0.8 \mathrm{M}_{\odot}$ stars. The adopted $[\mathrm{Fe} / \mathrm{H}]$ values are from Carretta et al. (2009). 
conductive opacities (from either Potekhin 1999 or Cassisi et al. 2007) or cross-sections for the ${ }^{14} \mathrm{~N}(p, \gamma){ }^{15} \mathrm{O}$ reaction (from either the NACRE compilation by Angulo et al. 1999 or the LUNA investigation by Formicola et al. 2004) are adopted. Encouragingly, the best agreement between theory and observations is obtained when the most recent physics is assumed. In fact, Marta et al. (2008) reported that the rate of this important ${ }^{14} \mathrm{~N}$ reaction should be reduced by a further $\sim 8 \%$, which would imply even better agreement with the data than that indicated by the curve labelled '(1)'.

The right-hand panel of Fig. 1 compares the predictions of fully up-to-date VictoriaRegina models (see VBD12) with the Bellazzini et al. findings for $\omega$ Cen and 47 Tuc. The close correspondence of the former with the latter must be fortuitous to some extent since, as shown in Fig. 2, the predicted core mass and the luminosity at the RGB tip are quite sensitive to the assumed chemical abundances (particularly of He and O). Although one expects that the helium abundance in metal-poor stars is close to the primordial value $(Y=0.2484 \pm 0.0016$; see Komatsu et al. 2011$)$, the oxygen abundance is quite uncertain. Indeed, since this element is primarily responsible for the differences between the curves for $[\alpha / \mathrm{Fe}]=0.0$ and $0.4 \mathrm{dex}$ (at fixed $Y$ ), the $\mathrm{O}$ abundance represents an important source of uncertainty in distance estimates that are based on the luminosity of the RGB tip. [The Victoria-Regina models predict slightly lower tip luminosities than the BASTI computations because the former (i) adopt the Marta et al. (2008) rate for the ${ }^{14} \mathrm{~N}(p, \gamma){ }^{15} \mathrm{O}$ reaction, (ii) take the gravitational settling of helium into account and (iii) assume somewhat different helium and metal abundances. (Diffusive models at low metallicities have reduced luminosities at the RGB tip by $\delta M_{\mathrm{bol}}=0.01-0.02$ mag.)]

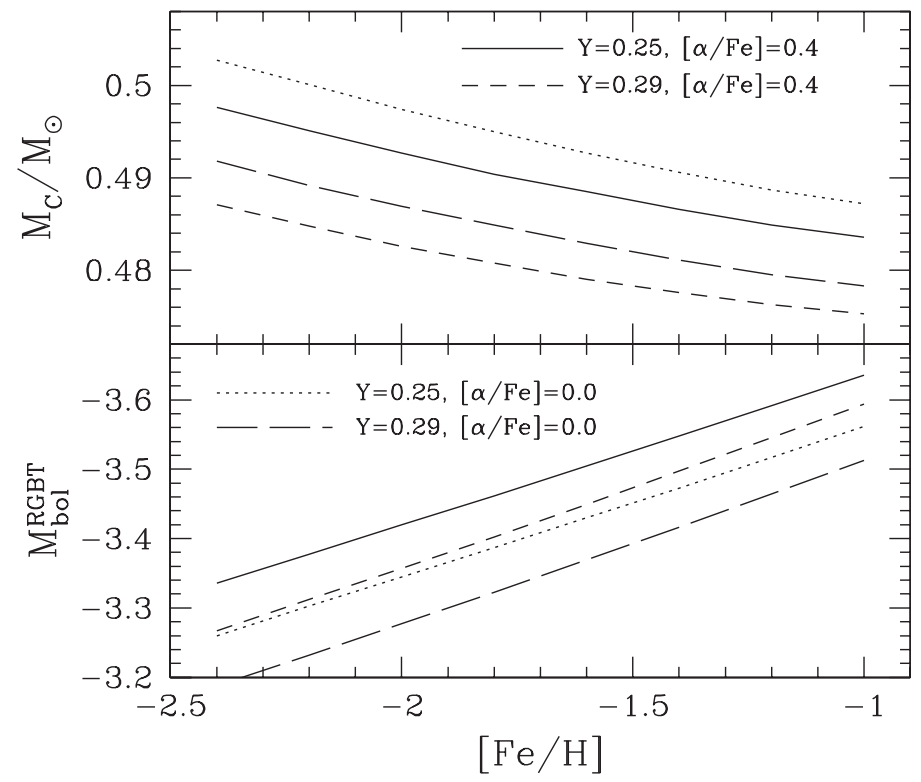

Figure 2. Dependence of the predicted luminosity (bottom) and helium core mass (top) at the RGB tip on $Y,[\alpha / \mathrm{Fe}]$ and $[\mathrm{Fe} / \mathrm{H}]$ according to the models of VBD12.

\section{The Luminosities of RR Lyrae Stars}

It is well-known that the luminosity of the horizontal branch varies inversely with metallicity and that, at a given $[\mathrm{Fe} / \mathrm{H}]$ value, it depends critically on the helium core 
mass $\left(\mathcal{M}_{\mathrm{c}}\right)$ and the envelope helium abundance. These dependences are illustrated in Fig. 3, which shows how the location of a theoretical zero-age horizontal branch (ZAHB) on the $(V-I)$ versus $M_{V}$ plane responds to changes in the abundances of iron, helium and oxygen, as well as to variations in $\mathcal{M}_{\mathrm{c}}$. (These results, which take into account the entire evolution from the main sequence to the RGB tip, were generated using the code described by VBD12.) The middle of the RR Lyrae instability strip is located at $(V-I) \approx 0.35$ mag: from studies of GCs that have $[\mathrm{Fe} / \mathrm{H}] \sim-1.6$ dex and relatively red HB populations (like M3), we know that the mean magnitude of their RR Lyrae stars is approximately 0.10 mag brighter than the ZAHB at the same colour (Sandage 1993).

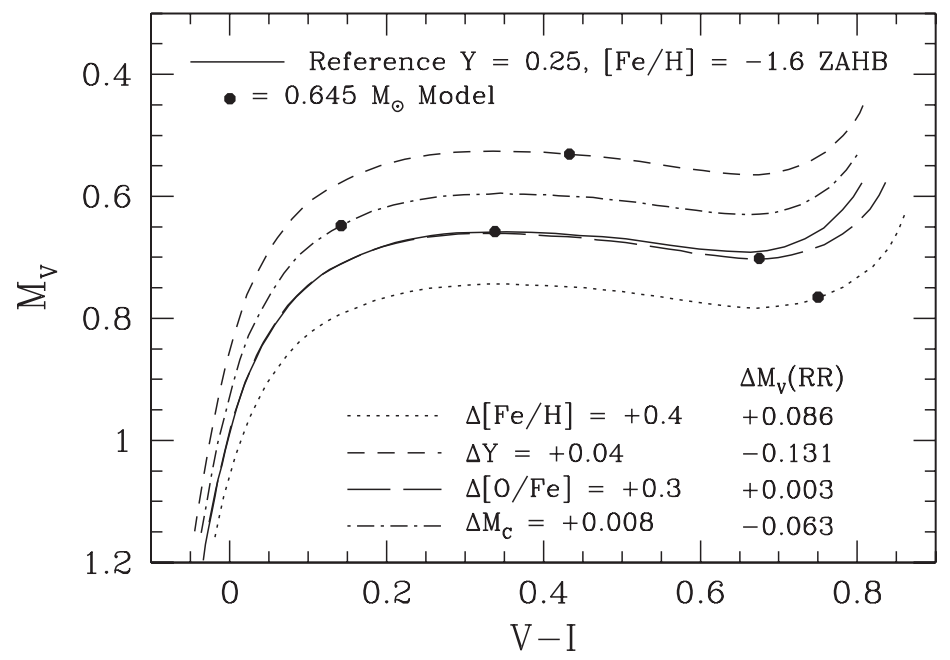

Figure 3. Plot on the $\left[(V-I), M_{V}\right]$ diagram of a computed zero-age horizontal branch (ZAHB) for $Y=0.25$ and $[\mathrm{Fe} / \mathrm{H}]=-1.60$ dex (solid curve), with additional ZAHBs that allow, in turn, for increased $[\mathrm{Fe} / \mathrm{H}], Y$ and $[\mathrm{O} / \mathrm{Fe}]$ abundances, as well as a somewhat larger helium core mass (as indicated). The effects of these variations on predicted RR Lyrae luminosities are listed in the bottom right-hand corner. The filled circles illustrate how the ZAHB location of a model for $0.645 \mathrm{M}_{\odot}$ depends on the values of the various parameters.

Although predicted turn-off luminosity versus age relations and the luminosity of the subgiant branch at a given age and colour strongly depend on the abundance of oxygen (see VBD12), the main effect of this element on the HB is to drive stars of a given mass to cooler temperatures (i.e. redder colours) with no more than a small impact on their luminosities; compare the locations of the filled circles on the solid and long-dashed curves in Fig. 3. This occurs, because an enhanced $\mathrm{O}$ abundance will tend to increase the relative importance of H-burning reactions over He-burning reactions. It is a wellestablished result that a ZAHB star will tend to be red if the H-burning shell is the main supplier of its luminosity, whereas it will be blue if most of its energy is provided by core He burning. By the same token, the recent reduction in the rate of the ${ }^{14} \mathrm{~N}(p, \gamma){ }^{15} \mathrm{O}$ reaction, and thereby in the relative importance of $\mathrm{H}$ burning, has the effect of shifting a ZAHB model to a higher $T_{\text {eff }}$ (by as much as $\delta \log T_{\text {eff }} \sim 0.05$; see Pietrinferni et al. 2010 ).

Although the luminosity of the HB, in the vicinity of the instability strip, has some dependence on $[\mathrm{O} / \mathrm{Fe}]$ at very low metallicities (see VandenBerg \& Bell 2001), O-abundance uncertainties are clearly inconsequential at $[\mathrm{Fe} / \mathrm{H}] \gtrsim-1.6 \operatorname{dex}$ (see Fig. 3). Thus, provided that the metallicity can be accurately determined from spectroscopy and that stellar models yield reliable helium core masses, the absolute magnitudes of RR Lyrae stars mainly depend on their helium abundances (and on their evolutionary states, since 


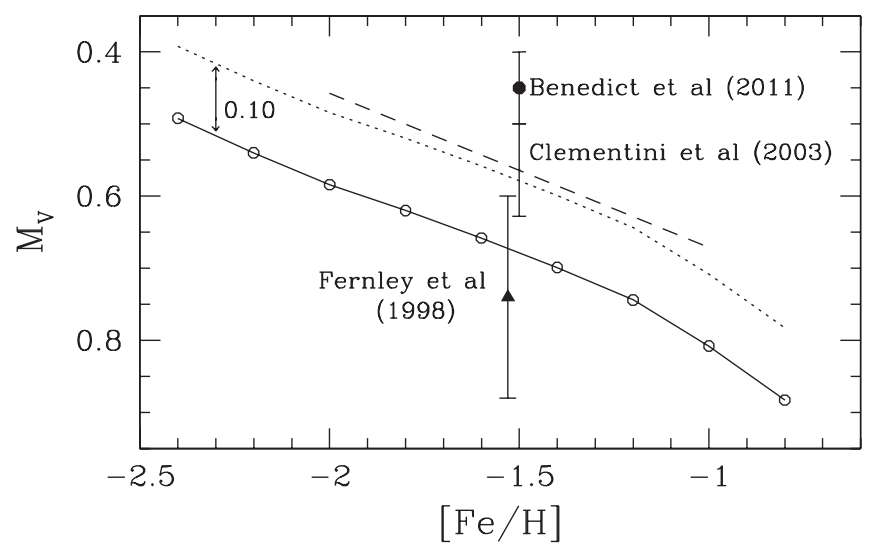

Figure 4. Comparison of the predicted $M_{V}-[\mathrm{Fe} / \mathrm{H}]$ relations for ZAHB models (solid curve and open circles) and for RR Lyrae stars (dotted curve), assuming that they are brighter, in the mean, by $0.10 \mathrm{mag}$ than the ZAHB, with the empirical relation (dashed line) derived by Clementini et al. (2003). The RR Lyrae $M_{V}$ zero points at $[\mathrm{Fe} / \mathrm{H}]=-1.5$ and -1.53 dex as determined from trigonometric parallaxes (Benedict et al. 2011) and from the Baade-Wesselink method (Fernley et al. 1998) are given by the filled circle and filled triangle, respectively.

they may have high luminosities if they have evolved into the instability strip from very blue or very red ZAHB locations). In this regard, it is especially important that the models treat diffusive processes, since - for fixed mass and chemical abundances - models that take this physics into account predict lower helium abundances at the RGB tip by $\delta Y \approx 0.013$ than those which do not. (They also predict lower values of $\mathcal{M}_{\mathrm{c}}$ by $\sim 0.003$ $\mathrm{M}_{\odot}$, which partially compensates for the reduction in $Y$.)

Based on an analysis of $>100$ RR Lyrae stars in the Large Magellanic Cloud (LMC), Clementini et al. (2003) obtained a mean $M_{V}=0.564 \pm 0.064 \mathrm{mag}$ at $[\mathrm{Fe} / \mathrm{H}]=-1.50 \mathrm{dex}$, for a true distance modulus of the LMC of 18.50. They also found that $\Delta M_{V} / \Delta[\mathrm{Fe} / \mathrm{H}]=$ $0.214 \pm 0.047 \mathrm{mag} \mathrm{dex}^{-1}$. These results, which are represented by the dashed line and the associated error bar in Fig. 4, may be compared with the dotted curve, which is the predicted mean luminosity of the RR Lyrae variables, assuming an offset of 0.10 mag from the ZAHB (the solid curve). (These models were also computed using the VBD12 code.) This is a valid comparison only at metallicities close to $[\mathrm{Fe} / \mathrm{H}]=-1.5$ dex, given that a somewhat smaller, or larger, offset between the ZAHB and the mean $M_{V}$ value for RR Lyrae stars is observed at lower, or higher, metal abundances (Sandage 1993). Parallaxes derived from Hubble Space Telescope/Fine Guidance Sensor observations obtained by Benedict et al. (2011) for five field RR Lyrae stars have yielded $M_{V}=0.45 \pm 0.05$ mag (the filled circle), which is just barely consistent with the Clementini et al. results. On the other hand, statistical parallax and Baade-Wesselink studies tend to favour relatively faint absolute magnitudes, as indicated by the filled triangle. The large uncertainty in the observed $M_{V}$ zero point means that RR Lyrae stars cannot be used (yet) to provide very tight constraints on either the distance scale or on the luminosities of theoretical models for $\mathrm{HB}$ stars. At least the slopes of the predicted and observed $M_{V}-[\mathrm{Fe} / \mathrm{H}]$ relations appear to be in good agreement.

\section{Subdwarf-based Distances}

Subdwarfs have been used to determine the distances to GCs since the beginning of the CCD era (e.g., Fahlman et al. 1985). However, despite improvements to both the 
parallaxes and the number of Population II dwarf standards that resulted from Hipparcos, there are still only about a dozen subdwarfs with $[\mathrm{Fe} / \mathrm{H}]<-1.2$ dex and $\sigma\left(M_{V}\right)<0.12$ mag that are not (obviously) binaries. Encouragingly, modern stellar models appear to be able to reproduce the properties of these stars in the $\left(\log T_{\text {eff }}, M_{V}\right)$ diagram, as well as many colour planes (see VandenBerg et al. 2010), provided that their temperatures and metallicities are close to the values derived or adopted by Casagrande et al. (2010). This is illustrated in Fig. 5: for this sample of 11 stars, the mean horizontal offset between the subdwarfs and the isochrones for their metallicities is only $25 \mathrm{~K}$ (with a standard deviation of $59 \mathrm{~K}$ ). Equivalently, the observed $[\mathrm{Fe} / \mathrm{H}]$ values and those of the isochrones on which the subdwarfs are located differ by only 0.11 dex in the mean (with a standard deviation of $0.27 \mathrm{dex})$.

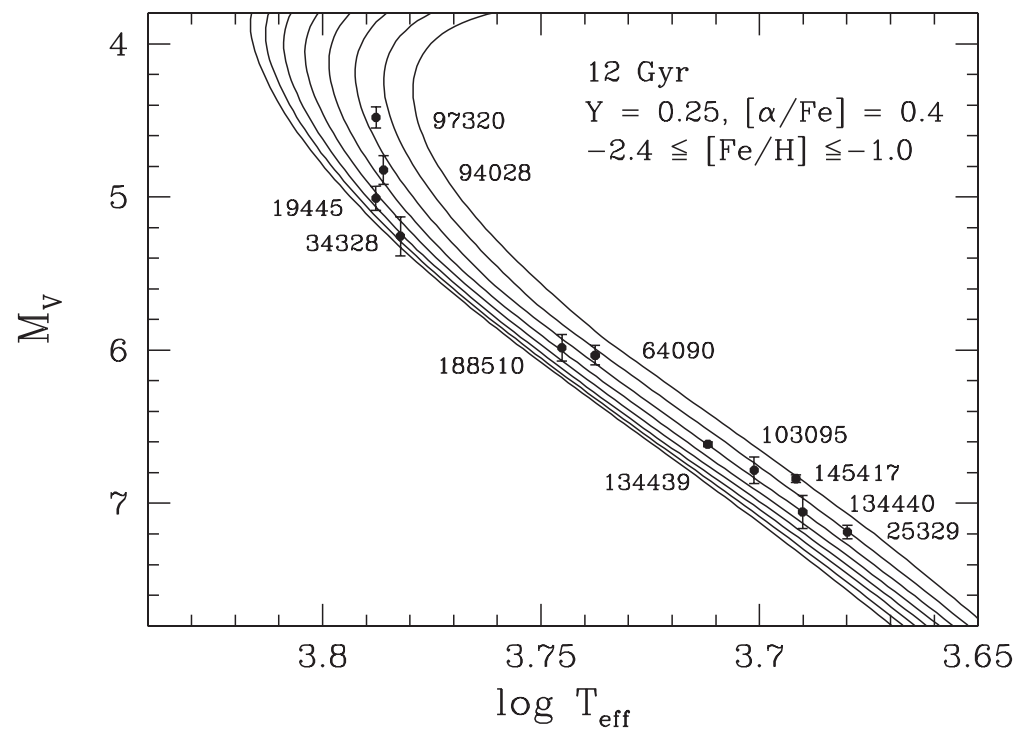

Figure 5. Superposition of local subdwarfs that have well-determined $M_{V}$ values from Hipparcos (van Leeuwen 2007) and temperatures based on the infrared-flux method (Casagrande et al. 2010) onto isochrones for the indicated age and $[\mathrm{Fe} / \mathrm{H}]$ values, in 0.2 dex intervals. The subdwarfs are identified by their HD numbers. The isochrones were computed by VBD12.

When performing main-sequence (MS) fits to local subdwarfs to derive the distances to star clusters or dwarf galaxies, errors in the target or subdwarf reddening values will clearly affect the results, as will differences in the helium abundances and/or the heavyelement mixtures. As shown by VBD12, variations in the abundances of $\mathrm{He}, \mathrm{O}, \mathrm{Ne}, \mathrm{Mg}$ and $\mathrm{Si}$ can have significant effects on the location of the MS, especially at $[\mathrm{Fe} / \mathrm{H}]$ values $\gtrsim-1.0$ dex. (At low metal abundances, isochrones are mainly affected by He and O.) Given the likelihood that all GCs harbour multiple, chemically distinct stellar populations (Carretta et al. 2010; and references therein), it cannot be assumed that field and cluster stars of the same $[\mathrm{Fe} / \mathrm{H}]$ have the same abundances of all other metals as well as helium. Indeed, field stars (with rare exceptions) do not show the O-Na anticorrelation that is characteristic of GCs. In addition, at a fixed $[\mathrm{Fe} / \mathrm{H}]$ value, they have $\mathrm{O}$ abundances that vary by $\sim 0.3-0.4$ dex as well as $\gtrsim 0.15$ dex variations in the abundances of many other metals (e.g., Ramírez et al. 2012). To improve upon current estimates of subdwarf-based distances, it will be necessary to determine whether the chemistries of the target stellar systems and each of the nearby calibrating stars differ in any significant way and, if there are important differences, to take them into account. 


\section{The Luminosity of the RGB 'Bump'}

Fusi Pecci et al. (1990) were the first researchers to use the luminosity of the RGB bump as a standard candle. ('Bump' refers to the local enhancement in the number of red giants at that luminosity where the H-burning shell passes through the chemicalabundance discontinuity that was produced as a result of the first dredge-up.) As in more recent studies (e.g., Di Cecco et al. 2010), they found approximately a 0.4 mag discrepancy between the predicted and observed bump luminosities in GCs when best estimates of the cluster distances were assumed. This feature is quite sensitive to the assumed chemical abundances - see Fig. 6-but even if models are generated for very high oxygen abundances (the dot-dashed curve), it is not possible to reconcile theory and observations, especially at the lowest metallicities. Overshooting of the convective envelope into the radiative interior (Alongi et al. 1991) may be the correct solution of this problem. In any case, the RGB bump is of limited use for distance determinations because of its considerable dependence on chemical abundances and on convection theory.

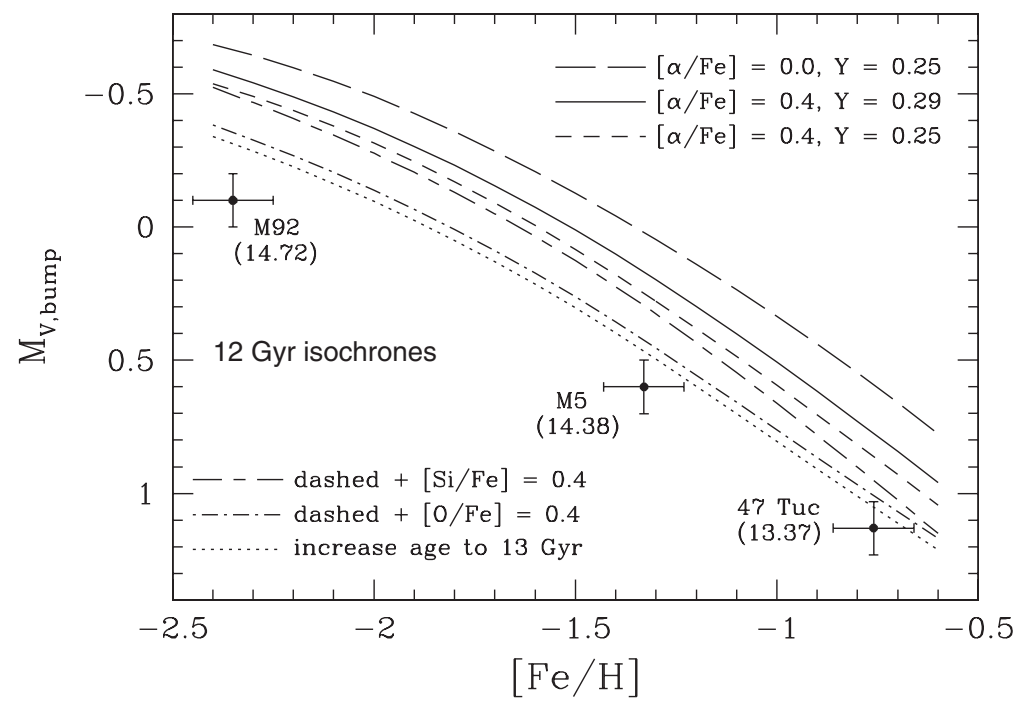

Figure 6. RGB bump luminosities as a function of $[\mathrm{Fe} / \mathrm{H}]$ predicted by 12 Gyr isochrones from the VBD12 study for the indicated helium and metal abundances. The observed $M_{V, \text { bump }}$ values for three GCs, for which the assumed apparent distance moduli are given in parentheses below their names, are from Di Cecco et al. (2010).

\section{Summary}

The distances to stellar systems can be derived to within $\delta(m-M)_{0} \lesssim 0.15$ mag using current empirical determinations of the RGB tip, RR Lyrae and subdwarf luminosities. The error bars are a reflection of the uncertainties in the distances and, owing in part to the $\sim 75 \mathrm{~K}$ uncertainties in the empirical $T_{\text {eff }}$ scale, the chemical abundances (especially of $\mathrm{He}$ and $\mathrm{O}$ ) of the calibrating objects. Valuable constraints on their chemical abundances may be obtained from eclipsing binary stars in open and globular clusters (e.g., Brogaard et al. 2012): this avenue of research needs to be fully exploited. Stellar models appear to be able to reproduce the properties of the standard candles quite well. Given recent advances, it seems unlikely that the physics of stellar interiors will undergo further significant revisions. However, it is necessary to improve upon our understanding of 
convection, the other mixing processes that affect surface abundances and the atmospheric boundary conditions. It is important to work towards the comparison of fully integrated 'atmosphere-interior' stellar models with observations of stars (including, in particular, the Population II subdwarfs in the solar neighbourhood).

\section{Acknowledgements}

I thank Santi Cassisi for providing the BASTI results that were used in Fig. 1 and the University of Victoria for the research grant associated with the recent Craigdarroch G. M. award, which supported this work.

\section{References}

Alongi, M., Bertelli, G., Bressan, A., \& Chiosi, C. 1991, A\& A, 244, 95

Angulo, C., Arnould, M., Rayet, M., et al. 1999, Nucl. Phys. A, 656, 3

Benedict, G. F., McArthur, B. E., Feast, M. W., et al. 2011, AJ, 142, 187

Bellazzini, M., Ferraro, F. R., Sollima, A., Pancino, E., \& Origlia, L. 2004, A\&A, 424, 199

Böhm-Vitense, E. 1958, Z. Astrophys., 46, 108

Brogaard, K., VandenBerg, D. A., Bruntt, H., et al. 2012, A\&3A, 543, A106

Carretta, E., Bragaglia, A., Gratton, R. G., D’Orazi, V., \& Lucatello, S. 2009, A\&3A, 508, 695

Carretta, E., Bragaglia, A., Gratton, R. G., Recio-Blanco, A., Lucatello, S., D'Orazi, V., \& Cassisi, S. 2010, A\&BA, 516, 55.

Casagrande, L., Ramírez, I., Meléndez, J., Bessell, M., \& Asplund, M. 2010, A\&A, 512, 54

Cassisi, S., Potekhin, A., Pietrinferni, A., Catelan, M., \& Salaris, M. 2007, ApJ, 661, 1094

Clementini, G., Gratton, R. G., Bragaglia, A., Carretta, E., Di Fabrizio, L., \& Maio, M. 2003, $A J, 125,1309$

Da Costa, G. S. \& Armandroff, T. E. 1990, AJ, 100, 162

Di Cecco, A., Bono, G., Stetson, P. B., et al. 2010, ApJ, 712, 527

Fahlman, G. G., Richer, H. B., \& VandenBerg, D. A. 1985, ApJS, 58, 225

Ferguson, J. W., Alexander, D. R., Allard, F., et al. 2005, ApJ, 623, 585

Fernley, J., Barnes, T. G., Skillen, I., et al. 1998, A\&A, 330, 515

Formicola, A., Imbriani, G., Costantini, H., et al. 2004, Phys. Lett. B, 591, 61

Fusi Pecci, F., Ferraro, F. R., Crocker, D. A., Rood, R. T., \& Buonanno, R. 1990, A\& A, 238, 95

Itoh, N., Uchida, S., Sakamoto, Y., Kohyama, Y., \& Nozawa, S. 2008, ApJ, 677, 495

Komatsu, E., Smith, K. M., Dunkley, J., et al. 2011, ApJS, 192, 18

Marta, M., Formicola, A., Gyürky, Gy., et al. 2008, Phys. Rev. C, 78, 022802

Nordlander, T., Korn, A. J., Richard, O., \& Lind, K. 2012, ApJ, 753, 48

Pietrinferni, A., Cassisi, S., \& Salaris, M. 2010, A\& A, 522, A76

Potekhin, A. Y. 1999, A\& A, 351, 787

Ramírez, I., Meléndez, J., \& Chanamé, J. 2012, ApJ, 757, 164

Richer, J., Michaud, G., \& Turcotte, S. 2000, ApJ, 529, 338

Sandage, A. 1993, AJ, 196, 719

Trampedach, R. \& Stein, R. F. 2011, ApJ, 731, 78

VandenBerg, D. A. \& Bell, R. A. 2001, New Astron. Rev., 45, 577

VandenBerg, D. A., Bergbusch, P. A., Dotter, A., Ferguson, J. W., Michaud, G., Richer, J., \& Proffitt, C. R. 2012, ApJ, 755, 15 (VBD12)

VandenBerg, D. A., Casagrande, L., \& Stetson, P. B. 2010, AJ, 140, 1020

VandenBerg, D. A., Edvardsson, B., Eriksson, K., \& Gustafsson, B. 2008, ApJ, 675, 746

van Leeuwen, F. 2007, A\&A A 474, 653 